\title{
"Mon corps est mon principal instrument de travail»
}

\section{Daniel Lüthi}

Journaliste et photographe indépendant, formateur en communication, Berne

L'hiver en Valais. La physiothérapie y est associée à des images familières: une activité débordante dans un cabinet high-tech reluisant de propreté, la musculation sur des appareils modernes, des thérapeutes en tenue blanche et au look sportif instruisant brièvement des victimes d'accidents de ski et de snowboard sur les exercices de réadaptation à accomplir. Ici, rien de tout cela - cette rencontre est toute de contrastes.

\section{Accordage affectif}

Le petit studio est difficile à trouver. Il se situe dans l'arrière-cour d'une maison de maître dans la vieille ville de Sion. Les deux pièces évoquent plutôt un appartement d'étudiant confortable que le lieu d'une activité clinique, les murs médiévaux sont épais, le plafond est porté par des poutres de bois sombres. La salle d'attente est peinte en orange méditerranéen soutenu, la salle de traitement est isolée avec des plaques de bois. Une plante et un tableau plutôt ténébreux d'une chouette y ont l'air casés ici faute de mieux - des cadeaux de patients. Quelques papiers épars et classeurs sans nom traînent çà et là, le regard par la fenêtre tombe sur un figuier derrière lequel se profile le chœur de la cathédrale toute proche. Au milieu de la pièce se dresse un lit réglable, une guitare est posée dans un coin. «Non, ce

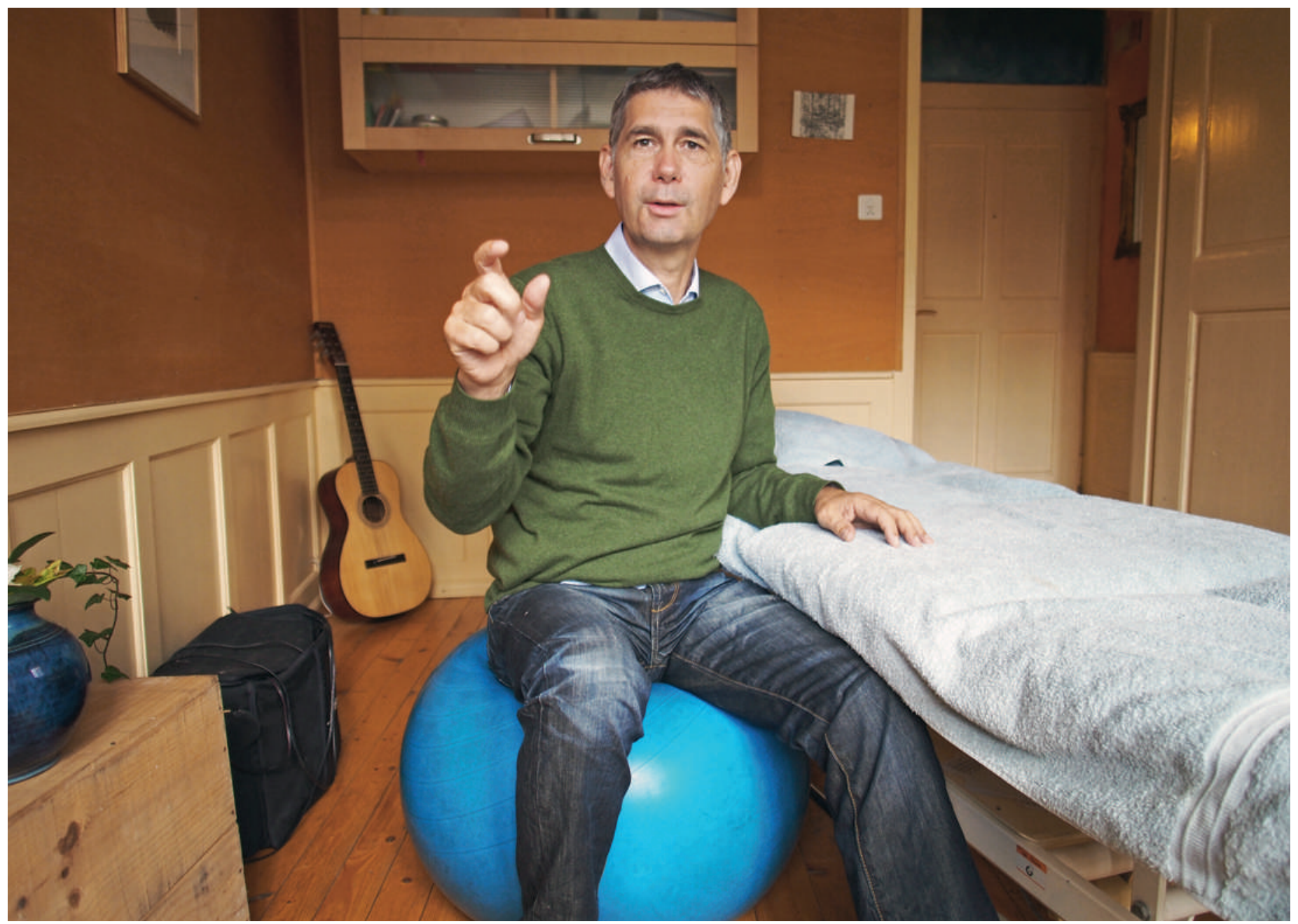


n'est pas un instrument de thérapie; elle sert à me détendre, à retrouver un équilibre pendant les pauses", dit Urs Daendliker.

On ne tarde pas à saisir le caractère éminemment symbolique de cette déclaration. Car même lorsqu'il parle de son approche thérapeutique, il pense d'abord à la manière dont il se sent: «Je connais l'âge, le métier et la pathologie du patient, mais seule ma propre expérience corporelle me renseigne sur ce qui se passe au moment précis. Rester à l'écoute de mon propre corps me donne des informations précieuses pour le moment décisif de l'établissement du contact.» Le psychanalyste Daniel Stern parle à ce propos d'un «accordage affectif», explique Daendliker. "L'écho de mon corps me signale la fatigue, la frustration ou le stress avant que je m'approche d'un patient et le touche.» Il se ménage donc, la marge nécessaire pour être lui-même avant de pouvoir transférer son sentiment sur le patient. «Une autorité, c'est quelqu'un qui autorise», dit Urs Daendliker. Sa philosophie: «Notre façon de penser et notre capacité d'analyse ne sont qu'une petite partie d'un système global. Mon principal instrument de travail est mon corps.»

\section{Une autre voie}

Bref, il n'a pas été question de gymnastique éprouvante sur des appareils sophistiqués, de point gâchette, d'électro- ou d'ultrasonothérapie, d'haltères, de bandes de fitness ou de trampolines. Le patient est invité à un voyage d'exploration de son corps. "Que ressentez-vous au genou?», demande-t-il par exemple à quelqu'un qui vient le voir après une opération du genou. «Est-ce comme s'il était en pierre - ou en bois? En métal, peut-être?» Il est intéressant d'écouter les images évoquées par un patient et d'observer ce qui

\section{«L'écho de mon corps me signale la fatigue, la frustration ou le stress."}

peut se déclencher par ce seul processus, dit notre interlocuteur, qui lui-même s'exprime volontiers en images. "Je suis comme un guide de montagne, je ne porte pas le sac à dos du patient et ne marche pas non plus à sa place.» Non, on ne peut vaincre un sommet ni guérir un genou par des seules paroles, répond Daendliker quand on lui pose la question, «mais le cheminement n'est pas direct. Quand vous avez mal, vous développez un système protecteur, un système fait de crainte et d'évitement. J'essaie d'aider le patient à trouver un nouvel accès à son corps.»

Sauf le respect que je lui dois, tout cela me paraît assez ésotérique. J’aurais des doutes sérieux si ce thérapeute

\section{Urs Daendliker}

Né à Nyon en 1961 d'un couple venu de Stäfa, Urs Daendliker a grandi à Nyon et à Genève. Sa maturité en poche, il a suivi l'école de physiothérapeutes à Genève, dont il est sorti diplômé en 1985 pour effectuer ensuite plusieurs formations postgrades: il a travaillé une année à I'hôpital pédia-

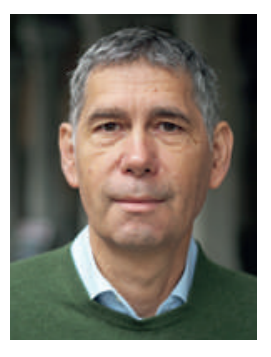

trique de Genève, s'est familiarisé avec la réflexologie, la sophrologie, la méthode Mézières et l'ostéopathie viscérale. En 1990/91, il a soigné des blessés de guerre pour le compte du CICR en Somalie et au Pakistan. II a ouvert son cabinet à Sion en 1992. Depuis, il ne cesse d'acquérir de nouvelles formations, par exemple en Médecine Traditionnelle Chinoise ou à la méthode Feldenkrais. Membre actif de l'association nationale physioswiss, il y collabore aussi au contrôle de qualité. Urs Daendliker est marié et père de deux enfants adultes. II vit avec sa femme à Champlan sur Sion.

ne m’avait pas été expressément recommandé par l'association officielle physioswiss. Alors poursuivons. Où sont par exemple les haltères? Ou les appareils qui permettent de remuscler un genou? «Un enfant ne touchera pas les haltères», répond clairement Daendliker sans se départir de son calme. «Un adulte pourra ou non être du même avis: s'il veut renforcer son genou, il utilisera peut-être à nouveau l'escalier au lieu de prendre l'ascenseur. Ce qui compte est la volonté d'y accéder, la confiance.» Suit une phrase déterminante: «Les appareils ne sont pas foncièrement une erreur. Ils représentent juste une autre voie que la mienne dans la prise en charge des patients.»

\section{Motion et émotion}

La physiothérapie moderne, Daendliker en est convaincu, continue de trop insister sur les aspects techniques: "L'accent est mis sur la volonté, la mesurabilité et le contrôle des performances», dit-il, ajoutant que la spontanéité du mouvement, l'imagination et la créativité sont trop souvent négligées. "Les aspects organiques tels qu'une inflammation, ou biomédicaux comme la force et la fonction sont une chose, mais ce qui compte pour moi est la participation. Par exemple, la question de savoir quand et comment un patient pourra se remettre au football.» Les exercices mécaniques sont alors moins importants que la conscience de pouvoir un jour réintégrer les mouvements dans sa vie quotidienne. «Beaucoup de méthodes qui nous aident dans cette voie ont été cédées à des professionnels non médicaux, comme des professeurs de tai chi ou de danse. Nous savons pourtant que tout ce qui nous remue au niveau émotionnel peut aussi nous faire bou- 
ger physiquement. S'émouvoir et se mouvoir sont très proches l'un de l'autre.» Par exemple, il peut être bon pour des personnes âgées souffrant de problèmes de hanche qu'elles se remettent à danser le fox-trot, la valse ou le cha-cha-cha comme dans leur jeunesse. «Ma qualité de physiothérapeute me donne un privilège précieux qui se raréfie dans notre culture», dit Daendliker, «celui de pouvoir toucher mes patients. Cela permet un échange non-verbal - et donne au patient la possibilité de rétablir le contact avec son propre corps et de reprendre confiance en lui.» L'une des spécialités dont se targue Daendliker est le toucher et la mobilisation en douceur de la peau du ventre, «une technique que j'ai apprise d'un ostéopathe français et qui produit des effets spectaculaires sur la respiration, mais aussi sur la mobilité de tout le torse». Il l'applique avec succès chez les enfants, les tétraplégiques, les bé-

\section{«Nous devrions avoir un accès direct à la population.»}

bés ou les mourants, par exemple. «Un grand nombre de mes collègues travaillent toujours dans le même segment», ajoute-t-il. "Ils s'en trouvent à jamais empêchés de développer comme il se doit la diversité de leurs compétences.» C'est vrai qu'il passe pour un oiseau rare dans son association, reconnaît Daendliker dans un sourire, «mais beaucoup de mes collègues me demandent de les traiter - ils savent bien pourquoi». Peut-être parce qu'ils savent qu'il forme des tuteurs pour les cercles de qualité. Mais peut-être aussi parce qu'il consacre une heure de temps à chaque patient et n'«expédie» pas plusieurs clients à la fois.

\section{La part de psychothérapie}

«Une patiente victime d'un accident tient d'abord à raconter comment il s'est passé», dit-il. «Une féministe m’a confié dernièrement qu'elle n'avait pas été heurtée par n'importe quel véhicule, mais par une jeep de l'armée. En théorie, ce détail est sans importance, mais dans la pratique, son poids peut être décisif. Le plus gros problème de cette femme n'était pas sa fracture du bras, mais un syndrome de stress post-traumatique.» Eh là, minute! Un physiothérapeute qui s'arroge des compétences de psychothérapeute? «Tous les thérapeutes sont habilités à écouter une patiente», répond-il, «et à l'accueillir comme un être humain à part entière.» Il est bien clair qu'il l'adressera à un spécialiste si nécessaire, "je sais reconnaître la ligne rouge». Cela dit, Daendliker s'insurge contre la règle générale qui veut qu'on ne puisse consulter un physiothérapeute que sur prescription médicale, c'est-à-dire qu'en cas de maladie objective. "Nous devrions avoir un accès direct à la population.» Une épaule "hors d'usage», par exemple, cache souvent une extrême tension ou une grande fatigue. «Si le patient se sent mieux après trois séances, c'est quand même le but, non?» Vus sous cet angle, les physiothérapeutes pourraient bien exercer une fonction de gatekeeper dans notre système de santé, Daendliker en est convaincu: «Nous pourrions même délester les cabinets médicaux des cas légers.»

Comment décrit-il sa relation avec les médecins? «D’après mon expérience, ce sont des gens très occupés et généralement sous pression. Leur responsabilité est énorme et ils ont à charge de tout objectiver et catégoriser. Quand je les contacte, j'ai souvent l'impression d'imposer au médecin une charge supplémentaire.» Contrairement à un médecin, lui-même n'est pas tenu de distinguer les niveaux objectif et subjectif, «je peux conclure une alliance totale avec le patient».

En sortant du cabinet, j'aperçois son logo: une silhouette exécutant une figure d'équilibre la tête dans les nuages, et non le regard vers le sol. «Une personne qui n’a pas le contrôle de la situation mais vit dans le présent», commente Urs Daendliker. "Qui marche mieux quand elle rêve. Qui danse dans un monde de rêve.» Effectivement.

\section{La prochaine "Rencontre avec...»}

A la fin de chaque mois, le Bulletin des médecins suisses présente une personnalité qui s'implique dans la santé publique. En mars, Daniel Lüthi parlera de sa rencontre avec Prof. Michael Thiel, médecin-chef de la clinique ophtalmologique de I'Hôpital cantonal de Lucerne. 